\title{
STATISTICAL PROPERTIES OF GRB AFTERGLOW PARAMETERS AS EVIDENCE OF COSMOLOGICAL EVOLUTION OF HOST GALAXIES
}

\author{
G. Beskin ${ }^{1}$, G. Oganesyan ${ }^{2}$, G. Greco ${ }^{3}$ and S. Karpov ${ }^{1}$
}

\begin{abstract}
The results of investigation of 43 peaked R-band light curves of optical transients of gamma-ray bursts with known redshifts are presented. The parameters of optical transients were calculated in the comoving frame, and then a search for pair correlations between them was conducted. As a result of the statistical analysis, a strong correlation between the peak luminosity and redshift was found for pure afterglows and the events with residual gamma activity, which can't be explained as an effect of observational selection. It suggests the cosmological evolution of the parameters of the local interstellar medium around the sources of gamma-ray burst. In the models of forward and reverse shock waves, a relation between the density of interstellar medium and redshift was built for gamma-ray burst afterglows. It was shown that for GRB host galaxies the star formation rate increases with redshift.
\end{abstract}

\section{Introduction}

Up to date, about 450 gamma-ray bursts (GRBs) with measured redshifts are known. The optical R-band light curves with distinct peaks were obtained for 43 cases only. These objects are the most interesting ones for a detailed analysis, as the presence of a peak allows us to identify the moment of the shock wave deceleration in the interstellar medium, which reflects the parameters of the interstellar medium. Among 43 such events, 11 are prompt optical peaks (P), coincident with gamma-ray activity (three events that may not be unambiguously classified as $\mathrm{P}$ were signed as P?), 22 are pure afterglows (A), and 10 more carry the signatures of an underlying gamma-activity $(\mathrm{A}(\mathrm{U}))$. Detailed results of investigation of correlations of different pairs of GRBs' parameters in these subsamples are given in Beskin et al. (2013). In this paper we present the analysis of connections between several optical characteristics of GRBs in source proper frame and their redshifts.

\footnotetext{
1 Special Astrophysical Observatory of the Russian Academy of Sciences, Russia

2 Southern Federal University, Russia

3 Astronomical Observatory of Bologna, INAF, Italy
} 


\section{Observational data}

The results of R-band optical observations, as well as other parameters of GRBs, were taken from the dedicated publications on specific bursts, GCN-circulars (http://gcn.gsfc.nasa.gov), and from the GRBLog database (http://grblog. org/grblog.php). The initial observational parameters were as follows: the spectroscopic redshift $\mathrm{z}$, the peak optical flux $\mathrm{F}_{\text {opt }}$, the integral optical flux $\mathrm{S}_{\text {opt }}$, the duration of optical emission $t_{\mathrm{opt}}$, the time of the peak onset relative to the GRB trigger $t_{\text {peak }}$, the width of the optical peak $t_{\text {width }}$, the exponents of growth and decay of the optical light curve $\alpha_{\mathrm{r}}$ and $\alpha_{\mathrm{d}}$, the GRB peak flux $\mathrm{F}_{\text {iso }}$, the GRB integral flux $\mathrm{S}_{\text {iso }}$, the GRB duration $\mathrm{t}_{90}$ and the photon index of the spectrum in the gamma-ray range $\alpha$.

Considering the galactic extinction and host galaxy brightness, and using the $\mathrm{k}$-correction for the average index of optical spectrum $\beta=0.75$ in the standard cosmological model with $\Omega_{\mathrm{M}}=0.3, \Omega_{\Lambda}=0.7, \mathrm{H}_{0}=70 \mathrm{~km} \mathrm{~s}^{-1} \mathrm{Mpc}^{-1}$, we obtained the following parameters in the proper frame of the source: the maximum optical luminosity $\mathrm{L}_{\mathrm{opt}}$, the isotropic equivalent of optical energy $\mathrm{E}_{\mathrm{opt}}$, the time parameters $\mathrm{T}_{\text {opt }}, \mathrm{T}_{\text {peak }}, \mathrm{T}_{\text {width }}$ and similarly in the gamma-ray range, $\mathrm{L}_{\text {iso }}, \mathrm{E}_{\text {iso }}, \mathrm{T}_{90}$. For the bursts whose host galaxy extinction $A_{R}$ are not available the mean value of $A_{R}$ was utilised instead, using the $A_{V}$ data collected in the "golden sample" presented by Kann et al. (2010). These data were divided into five redshift ranges and for each interval the corresponding mean value of $A_{V}$ was obtained. Using these estimates along with the dependence of absorption on wavelength in SMC (Pei 1992), the $A_{R}$ for each burst was computed.

The formulae for conversion from the observed frame to the proper one are taken from Beskin et al. (2013). Table 1 presents all pair correlations with unweighted Pearson correlation coefficients $\mathrm{R}>0.5$ and significance levels $\mathrm{SL}<1 \%$, and the coefficients of corresponding linear regressions.

\section{Results and discussion}

To prove that the correlation between peak optical luminosity and the redshift is not caused by the selection effects, we plot in the right panel of Figure 1 the R-band apparent magnitudes of all bursts at the initial moment of optical detection (empty symbols) and at the moment of maximum (filled symbols) versus redshift. Note that the signatures of selection effects should be searched for in the set of initial brightness estimates at the first place. Let's discuss whether they are present in our data.

1. Obviously, if the proper luminosities of sources do not increase with redshift (i.e. luminosity is the same on all $\mathrm{z}$ ), then apparent brightness (flux measured by the observer) will decrease at least quadratically with $(1+\mathrm{z})$. At the same time, right panel of Figure 1 demonstrates significant increase of the brightness at $\mathrm{z}>3$, both for the moments of detection and for the peaks.

2. For both large $(\mathrm{z}>3)$ and small $(\mathrm{z}<1)$ redshifts the objects are brighter than 18 mag, significantly brighter than the minimum value of 19-19.5 mag achieved by several objects at $1<\mathrm{z}<3$. Therefore, neither small nor large $\mathrm{z}$ 
Table 1. Pair correlations for different classes of GRB optical counterparts with the correlation coefficients greater than 0.5 and significances better than $1 \%$. The four columns represent the linear regression $(\mathrm{a}+\mathrm{bx})$ coefficients, derived through the unweighted least squares fit. The stars mark the log-linear correlations, in contrast to the log-log correlations used otherwise.

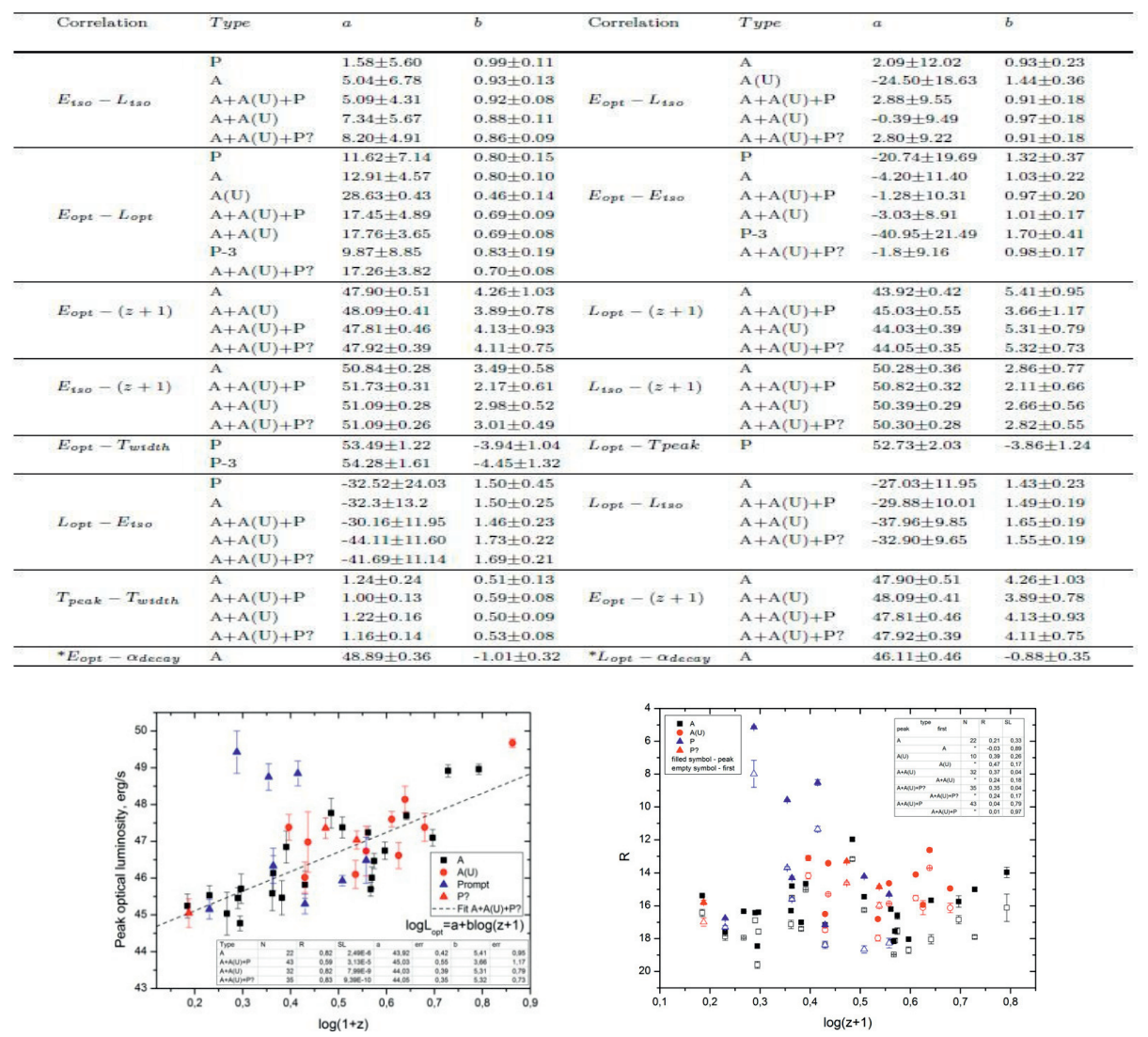

Fig. 1. Left: peak optical luminosity vs. redshift: coefficients of correlations, SL, parameters of linear regression. Right: peak and initial optical magnitudes vs. redshift: coefficients of correlations, SL.

display any signs of the bias due to crossing the detection limit line - both bright and faint sources are being detected on all redshifts.

3. Finally, returning to $\mathrm{L}_{\mathrm{opt}}-(\mathrm{z}+1)$ dependence (left panel of Fig. 1), we checked the correlation coefficients and linear regression parameters for $\mathrm{A}+\mathrm{A}(\mathrm{U})+\mathrm{P}$ ? subset in different redshift ranges. They are summarised in Table 2 .

It is easy to see that even with exclusion of objects with $\mathrm{z}<1$ or with $\mathrm{z}>4$ or both, the luminosity still increases with redshift with good significance. Obviously, the correlation coefficient decreases a bit with decreasing of redshift range, but the 

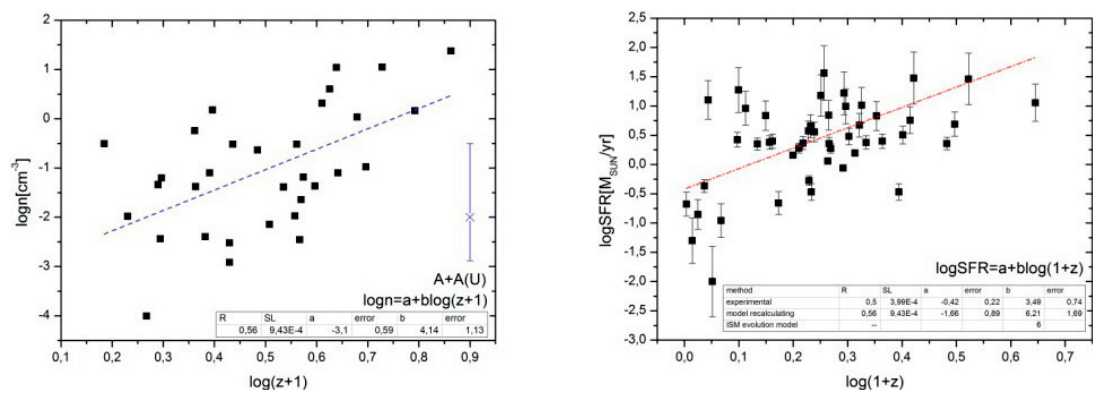

Fig. 2. Left: ISM density vs. redshift by model recalculation. Right: SFR vs. redshift correlation according to GRBHosts (http://grbhosts.org) database.

Table 2. Characteristics of the dependence of optical luminosity on redshift for $\mathrm{A}+\mathrm{A}(\mathrm{U})+\mathrm{P}$ ? subset in different redshift ranges. Columns are the redshift range, number of sources in it, correlation coefficient, its significance level, and the linear regression parameters $(\mathrm{a}, \mathrm{b})$ with corresponding errors.

\begin{tabular}{|c|c|c|c|c|c|c|c|}
\hline $\mathrm{z}$ & $\mathrm{N}$ & $\mathrm{R}$ & SL & a & err & b & err \\
\hline all & 35 & 0,83 & $9,39 \mathrm{E}-10$ & 44,05 & 0,35 & 5,32 & 0,73 \\
\hline $\mathrm{z}<4$ & 32 & 0,73 & $1,73 \mathrm{E}-6$ & 44,29 & 0,37 & 4,71 & 0,8 \\
\hline $\mathrm{z}>1$ & 31 & 0,78 & $1,91 \mathrm{E}-7$ & 44,12 & 0,5 & 5,2 & 0,97 \\
\hline $1<\mathrm{z}<4$ & 28 & 0,6 & $7,31 \mathrm{E}-4$ & 44,55 & 0,55 & 4,22 & 1,11 \\
\hline
\end{tabular}

regression parameters are nearly the same within the errors, and the power-law slope of the dependence is roughly $4-5$.

Therefore, our analysis demonstrates that effects of observational selection which may cause the dependence of optical luminosity in peaks of light curves on redshift, are most probably absent in our data.

Therefore, we may consider the detected $\mathrm{L}_{\mathrm{opt}}-(\mathrm{z}+1)$ correlation (left panel of Fig. 1) as a real manifestation of evolution of optical luminosity of gamma-ray bursts.

There is no $\mathrm{L}_{\mathrm{opt}}-(\mathrm{z}+1)$ correlation for prompt optical sources in contrast to a strong correlation seen for afterglows. The prompt optical sources $(\mathrm{P})$ are presumably produced as a result of collisions of internal shells in GRB sources, while the afterglows are formed as the shock wave enters the interstellar medium. Correspondingly, the $\mathrm{L}_{\mathrm{opt}}-(\mathrm{z}+1)$ correlation for the afterglows points to the dependence of the interstellar medium parameters on redshift. The simplest assumption is a dependence of the local interstellar medium density on redshift, which results in the observed $\mathrm{L}_{\mathrm{opt}}-(\mathrm{z}+1)$ dependence.

In the afterglow model with the front shock wave, the peak flux is a function of density, as we may assume that the frequency of optical emission lies between the characteristic frequency of radiation and the cooling frequency. Indeed, according to Panaitescu (2009), if the frequency of the afterglow spectral peak $v_{i}$ is lower than the cooling frequency $v_{c}$, then the optical spectrum index is $\beta=\frac{p-1}{2}$, where $\mathrm{p}$ is a spectral index of emitting electrons, $p>2, p=2 . .3$ (Piran 2004), and $\beta=0.5 . .1$. 
If, on the other hand, the peak is in the $v>v_{c}$ region, then $\beta=\frac{p}{2}=1 . .1 .5$. At the same time, observations of optical spectra give $\beta<1$ (Zafar 2011) with average value of $\beta=0.75$, and therefore our assumption is correct. Then, according to Panaitescu et al. (2011),

$$
\mathrm{F} \propto \operatorname{En}^{\beta+1 / 2} \Gamma_{0}^{4 \beta}
$$

where $\mathrm{E}$ is the total mechanical energy, $\mathrm{n}$ is the volume density of the surrounding gas, $\Gamma_{0}$ is the initial Lorentz factor of the ejecta, and $\beta$ is the index of optical spectrum. Using $\Gamma_{0}=200$ with the dispersion of 100 , the peak luminosity $\mathrm{L}_{\mathrm{opt}}, \mathrm{E}_{\mathrm{iso}}=\eta \mathrm{E},(\eta=0.2-$ from Sari et al. 1999), and the deceleration radius $\mathrm{R}_{\mathrm{dec}} \propto \mathrm{t}_{\mathrm{dec}} \Gamma_{0}^{2}$ (Meszaros 2006), where $\mathrm{T}_{\text {peak }}=\mathrm{t}_{\mathrm{dec}}, \mathrm{L}_{\mathrm{opt}} \propto \mathrm{R}_{\mathrm{dec}}^{2} \mathrm{~F}, \eta \propto \frac{L_{o p t}}{T_{\text {peak }}^{2} E \Gamma^{5}}$, we obtain power-law $n-(z+1)$ dependence with slope of $4.14 \pm 1.13$ (Fig. 2, left panel). With this dependence in hand, we may built a similar dependence for the star formation rate (SFR) in the GRBs vicinity using the Kennicutt-Schmidt law from Schaye et al. (2007): the star formation rate depends on the volume density of the interstellar medium as SFR $\propto \mathrm{n}^{1.5}$. Finally, we acquired SFR $\propto(\mathrm{z}+1)^{6.21 \pm 1.69}$.

Using the values of SFR taken from the GRBHosts (http://grbhosts.org) database, we have compared them to this relation. This is shown in Figure 2 (right panel), which also shows the model value of this dependence based on the ratios for the interstellar medium density from Ciardi et al. (2000) and the volume law of Kennicutt-Schmidt.

The obtained SFR - $(z+1)$ connection is consistent with the model dependence from Ciardi et al. (2000), but differs from the experimental correlation for the host galaxies from the GRBHosts (http://grbhosts.org) database. Presumably, this can be explained by the fact that our estimations of SFT are applied to small zones of GRB localisation, but not to the galaxies as a whole.

This work was supported by the RFBR No. 12-02-00743), and by the grant of the President of the Russian Federation for the support of young Russian scientists. S.K. has also been supported by a grant of the non-profit Dynasty foundation. G.B. thanks Landau Network-Cenro Volta and Cariplo Foundation for fellowship and the Brera Observatory for hospitality.

\section{References}

Beskin, G., Greco, G., Oganesyan, G., et al., 2013, AP, 53, 5

Ciardi, B., \& Loeb, A., 2000, ApJ, 540, 687

Kann, D.A., Klose, S., Zhang, B., et al., 2010, ApJ, 720, 1513

Meszaros, P., 2006, IOP, 69, I8, 2259

Panaitescu, A., 2009, Sixth Huntsville Symposium, AIP Conference Proceedings, 1133, 127

Panaitescu, A., \& Vestrand, W.T., 2011, MNRAS, 414, I4, 3537

Pei, Y.C., 1992, ApJ, 395, 130

Piran, T., 2004, RvMP, 76, I4, 1143

Sari, R., \& Piran, T., 1999, ApJ, 520, 641

Schaye, J., Dalla, V.C., 2008, MNRAS, 383, 1210

Zafar, T., Watson, D., Fynbo, J.P.U., et al., 2011, A\&A, 532, id.A, 143 
